\title{
Study on Different Extraction Methods of Gomphocerus Sibilants Genomic DNA
}

\author{
Jinxiu Mu \\ Weifang University of Science \& Technology, Shandong, Shouguang, 262700, China.
}

Keywords: Genomic DNA extraction;Different extraction methods;Gomphocerus sibiricus; Experimental

\begin{abstract}
In the experiment, the gras shopper in Siberia is taken as the research objec. The comparison of the genomic DNA of dried and soaked in alcohol samples are extracted by the method of saturated $\mathrm{NaCl}, \mathrm{CTAB}$ and SDS. Experimental results show that SDS method brings the total DNA, saturated Nacl law and CTAB extraction law of total DNA in specimens of the lower rate in most Agarose gel electrophoresis testing marked degradation. In addition, ethanol college system obtained by overall DNA specimens is higher in the rate and quality of than that of the dry specimens.
\end{abstract}

\section{Introduction}

The locust is one of the main pests of agriculture, animal husbandry and forestry, and it is necessary to identify the species of locust effectively. There have been a great many reports which are about locusts classification research. The predecessors collected a large number of specimens, named a large number of pattems which constitute the basis and framework of grasshopper taxonomy, molecular biology technology research of locusts taxonomy and evolution. Using these specimens and mode can greatly extend the grasshopper taxonomy and evolution of molecular biology research. The extraction of high quality and high yield genomic DNA are the basis and prerequisite for molecular biology studies.

\section{Materials and methods}

Experimental materials. Locust specimens, extraction reagent, electrophoresis reagent. The main instrument: bench top high speed refrigerated centrifuge, electronic balance, seat type au tomatic steam sterilization pot, hot air sterilizer, electro thermostatic water bath, super clean bench, refrigerator electrophoresis, UV analyzer and so on.

Research method. SDS- proteinase K digestion

1) Sampling: Locust stem specimens and anhydrous ethanol impregnated specimens with sterile distilled water rinse for $2 \sim 3$ times, soaked in sterile distilled water for more than 48 hours, waste water, discarding the soaking liquid, locust hind femur muscle, shredded material to $0.8 \mathrm{ml}$ of homogenate (a liquid) $1.5 \mathrm{ml}$ Eppendorf tubes filled with.

2) Grinding: the glass rod matched with the centrifugal tube will be filled with the homogenate of the sample..

3) Add $0.15 \% \mathrm{SDS} \mathrm{mL}$ and $2 \mathrm{mg} / \mathrm{mL}$ of $0.1 \mathrm{~mL}$ protease $\mathrm{K}$.

4) Water bath: $37^{\circ} \mathrm{C}$ water bath $1 \sim 3 \mathrm{~h}$, until the mixture is very clear so far to digest.

5) Phenol extracts: in the mixed liquid, such as the size of the balance phenol, the upper and lower slowly upside down ten times, do not violent oscillation, on the desktop high-speed centrifuge 6000 $\mathrm{r} / \mathrm{min}$ centrifuge $10 \mathrm{~min}$, supernatant.

6) Repeat step 5: until the interface of the aqueous phase and phenol phase does not appear white protein layer, supernatant. 
7) Chloroform: isoamyl alcohol (24: 1) extraction: the supematant with the addition of an equal volume of chloroform: isoamyl alcohol, slowly reversed ten times, in high speed tabletop centrifuge 8 $000 \mathrm{R} / \mathrm{min}$ centrifugation for $10 \mathrm{~min}$, the supernatant. Repeat this step.

8) Precipitation DNA

9) Washing DNA

10) Save: natural drying, adding TE or sterile distilled water dissolved in three,. $20^{\circ} \mathrm{C}$ to preserve the reserve.

Saturated $\mathrm{NaCl}$ method.

1) Ethanol fixed specimens soaked in sterile distilled water for 48 hours; specimens of dry, soak (TE) soaked for more than 48 hours, discarding the soaking liquid to, after the removal of the locust foot thigh muscle.

2) Cut the material, add 4001 digestive juices at 55 degrees fordigestion of 8 to $12 \mathrm{~h}$ above, and then protease $\mathrm{K}(2 / \mathrm{mL} \mathrm{mg}), 37$ degrees continue to digest $1 \mathrm{~h}$.

3) $300 \mathrm{~L}$ saturated $\mathrm{NaCl}$, scroll $30 \mathrm{~s}, 10000 \mathrm{r} / \mathrm{min}$ centrifugal $30 \mathrm{~min}$.

4) The transfer supernatant, ISO volume ISO alcohol precipitation DNA, 1000r/min centrifugal $15 \mathrm{~min}$, Kami Kiyo.

5) $70 \%$ ethanol wash DNA precipitation, dry naturally, TE $(\mathrm{pH} 8)$ after dissolution, at $-20^{\circ} \mathrm{C}$ for reserve.

CTAB

1) The locusts on dry specimens and anhydrous ethanol soaked specimens with sterile distilled water washed $2 \sim 3$ times; soaked in sterile distilled water for more than 48 hours, waste water, discarding the soaking liquid, locust hind femur muscle and speed put into EP tube crush.

2) Adding $2 *$ CTAB buffers $300 \mathrm{~L}$ to 65 temperatures of $60 \mathrm{~min}$. Then, $12000 \mathrm{r} / \mathrm{min}$ centrifugation of $15 \mathrm{~s}$, the supernatant was poured out.

3) The original centrifuge tube in the remaining tissue adds $200 \mathrm{mu} \mathrm{L} 2 *$ CTAB buffers and gently whisk, after $65 \mathrm{DEG} \mathrm{C}$ incubated for $10 \mathrm{~min}, 1000 \mathrm{r} / \mathrm{min}$ centrifugation for 10 minutes, pour out the supernatant and the first collected supernatant was mixed.

4) The CI was extracted with equal volume of, $10000 \mathrm{r} / \mathrm{min}$ centrifugation $10 \mathrm{~min}$, and supernatant was extracted..

5) Adding ice cold $\mathrm{r} / \mathrm{min}$ precipitation, 8000 min centrifugal 10. Precipitation, plus $70 \%$ ethanol $200 \mu \mathrm{L}$, washing DNA precipitation, $8000 \mathrm{r} / \mathrm{min}$ centrifugal $6 \mathrm{~min}$, pour supernatant.

6) Natural drying, adding TE or sterile distilled water dissolved in three. $-20^{\circ} \mathrm{C}$ to preserve the reserve.

\section{Electrophoresis results and analysis}

Electrophoresis. Agarose gel electrophoresis detection:

Reagents: Agarose, bromide ethidium staining solution, 1 x 5TBE: Tris 54G, boric acid 27.5g, 0.5 mol / L EDTA 20ml (pH 8.0), with triple distilled water set to $1 \mathrm{~L}$, sterilization and storage, 10 times diluted concentration.

Loading buffer:0.25\% bromophenol blue and $40 \%$ sucrose, were preserved in three distilled waters.

Adhesive:

1) Seal the glue through and place the level.

2) $0.4 \mathrm{~g}$ agarose plus $40 \mathrm{ml} 1 * 0.5 \mathrm{TBE}$ after heating to dissolve bright shake.

3) Rubber is cooled to 65 DEG C to pour the gel. The tank thickness is about $5 \mathrm{~mm}$ with inserted comb. It is placed at room temperature for $40 \mathrm{~min}$, until the gel completely and carefully remove the comb.

4) In the electrophoresis tank, the gel tank is added to the surface of the gel buffer.

Sample DNA $5 \mu 1$ and $2 \mu l$ of bromine phenol blue after mixing with a moving liquid gun point into the hole, note that the sample before mixing. 
Electrophoresis: Cover the electrophoresis tank and pass the electricity which is $120 \mathrm{~V}$ constant pressure electrophoresis of 40-60 min.

Staining: agarose gel was placed in EB $15 \mathrm{~min}$.

Observation: place in the ultraviolet analyzer, and then with a gel imaging system, observe the camera.

Total DNA extraction results. Fig. 2 , Fig. 1 and Fig. 3 are the Siberia locust genome DNA 1\% ag aro se gel electrophoresis chart.

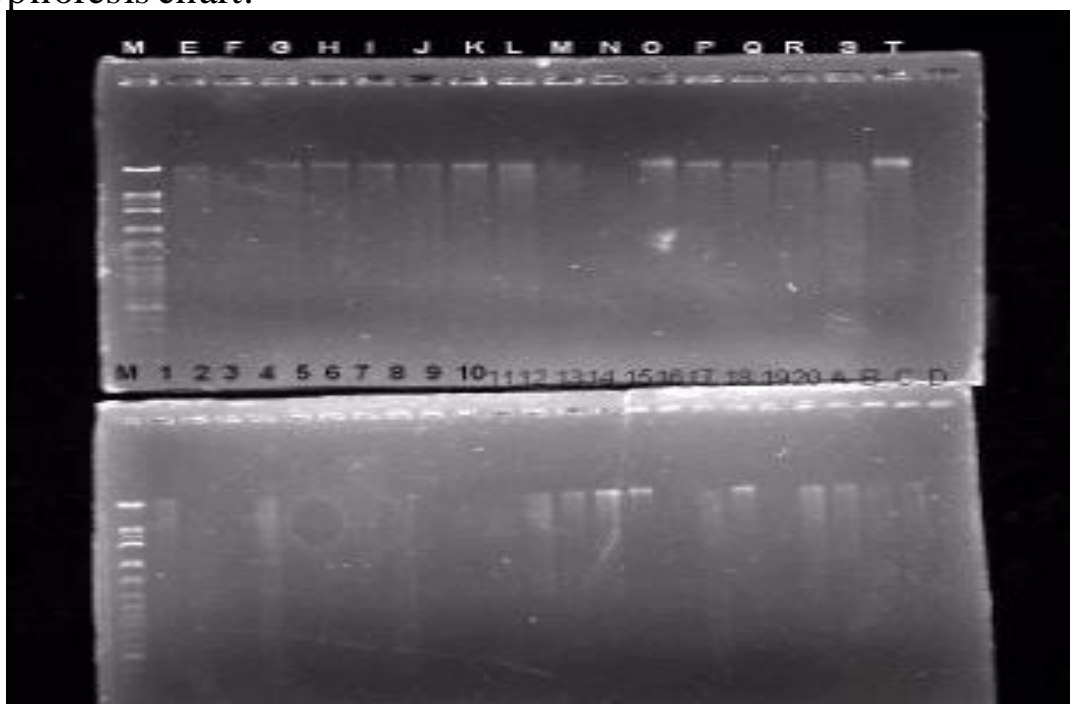

Fig. 1 DNA K agarose gel electrophoresis chart was extracted by SDS- protease digestion

\section{MABCDEFGH LIKM}

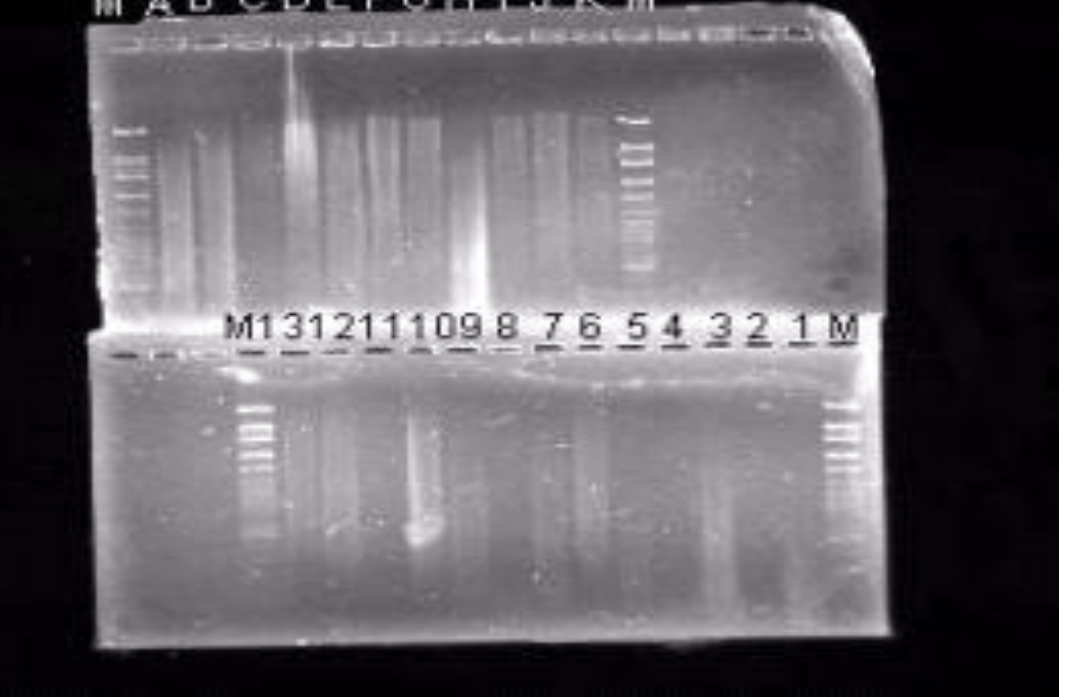

Fig. 2 DNA agarose gel electrophoresis chart was extracted by saturated $\mathrm{NaCl}$ method 


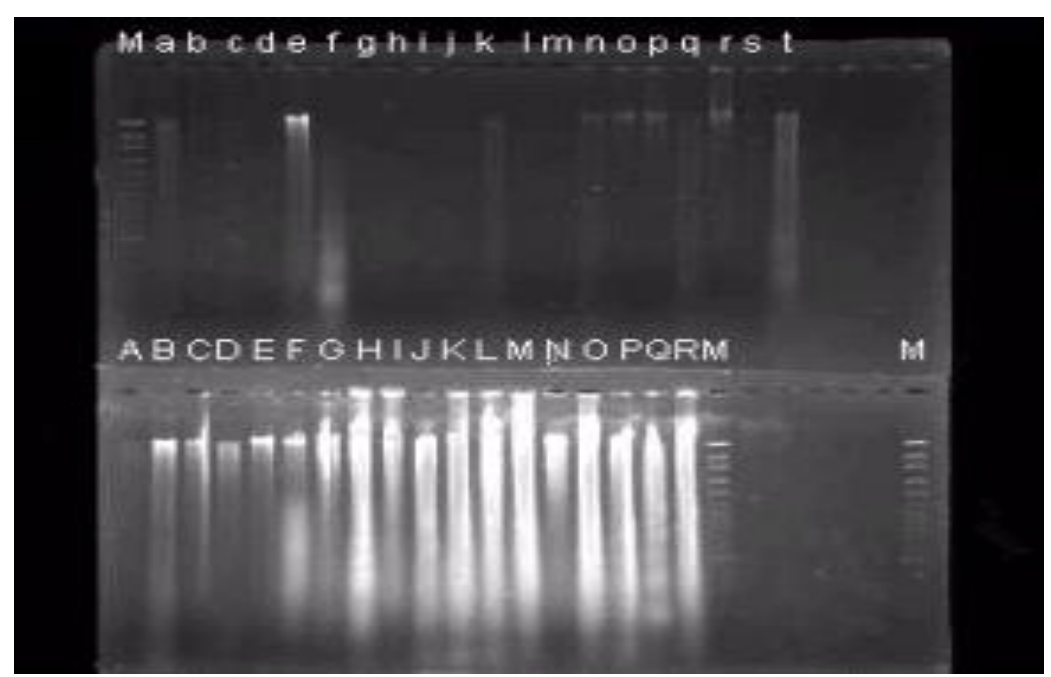

Fig. 3 Agarose gel electrophoresis of DNA by CTAB

Results and analysis. Generally, after electrophoresis, if the sample DNA is strip neat but not tail, it indicates that DNA is more complete which is not impurities. Conversely, the DNA sample is considered to degrade or mixed with impurities. It was found that samples of this experiment have obvious DNA bands, but they have different degradation.

Fig. 1 shows that SDS proteinase K digestion method is used to extract total DNA in the 3000bp with a bright band. If the basic is not tail, it indicates that the extraction of DNA molecules are more complete. Saturated $\mathrm{NaCl}$ method is to extract total DNA. From Fig. 2, it can be seen that the effect of using this method to extract is relatively poor. DNA dispersion is larger, which does not appear single with the concentration. The wipe trace phenomenon is serious. Even this method is used to extract genomic DNA, it can be amplified by PCR. DNA is extracted by CTAB. method. From Fig. 3, it can be seen that stem from only one sample makes a clear DNA bands. Specimens of anhydrous ethanol immersion extraction of total DNA have bright band in $3000 \mathrm{bp}$, but most of them have a tail. And tailing phenomenon is more serious, indicating that the sample DNA degradation is mixed with impurities. The results of the three methods shows that the effect of SDS- protease K digestion is the best. The CTAB is the second, and the saturated $\mathrm{NaCl}$ is not effective.

\section{Analysis of the reasons for the unsatisfactory results}

1) Because of climate reasons, fresh specimens are not adopted. The specimens and ethanol fixed specimens are stored for too long to severe DNA degradation (DNA is chemically unstable which can be hydroly sis or oxidation spontaneous degradation),

The extraction effect is not better than that of fresh specimens;

2) The raw material is too little and the grinding is not enough;

3) During the mixing of the drugs or the blowing process, the action is violent or the head of the gun is too small to cause the breaking of the DNA frag ment;

4) In the SDS- protease K digestion, the protein might not be digested completely;

5) Three extraction and chlorine of phenol in the process of SDS- protease K digestion and CTAB

The simulated extraction of ethanol may not be enough or the time of the cen trifugal rotation speed is insufficient, so that the supernatant fluid contain s more impurities, which affect the purity of DNA.

In addition, during the extraction of genomic DNA, the integrity of the nucleic acid primary structure should be ensured, and the pollution can be reduced. Therefore, in addition to simplify the operation steps, to shorten the extraction process, to avoid making the nucleic acid in high temperature, acid or alkali environment, to reduce the chance of nucleic acid deg eneration, in the extraction process, it is also necessary for the experiental techniques to minimize the influence of chemical and mechanical factors. 


\section{Conclusions}

Since the purity of the concentration of DNA is good, SDS- protease K digestion method can be widely used. The DNA method proposed by saturation with $\mathrm{NaCl}$ contains more impurities, so the method can beused for molecular experiments on the high quality of the template. The CTAB method is suitable for the extraction of DNA of genomic of small insects, and the supernatant is only two times and the organic extract is once, and the DNA was high.

\section{References}

[1] LENNEYC, WILL IAMS S L, GOLDSON D B, et al. Geographical orig in of an introduced insect pest, Listronotus bonariensis( Kuschel), determined by RAPD analysis[J] . Heredity , $1994,72: 412-419$.

[2] Zhemin Zheng. Locust taxonomy [M]. Xi'an: Shaanxi Normal University press, 1993.

[3] Yaping Guo, Zhemin Zheng, Enbo Ma. Cytotaxonomical China Oxya [J]. Chinese Insect Science (UK), 1994, 1 (2): 101-109.

[4] Yihao Duan, Enbo Ma, Haixuan Qiao. A comparative study of some species of Acridoidea allozy me (En glish) [J]. The Journal of genetics, 29, 2002 (2): 133-137.

[5] Peijie Dai, Dazhi Zhang. A study on the extraction of DNA from the insect [J]. Joumal of Ning xia agricultural college, 25, 2004 (3): 10-13.

[6] Jianzhen Zhang, Yaping Guo, Enbo Ma. DNA extraction and RAPD analysis of locust tissues under different preservation methods [J]. Journal of zoology, 2004, 39 (2): 53-57.

[7] Weizhi Ji, Bing Su. The principle and method of genetic diversity [M]. Hangzhou: Zhejiang Science Press, 1999, 61-62.

[8] Zhujun Tan, Yali Han,Hefeng Su. A comparative study of six species of Chorthippus genomic DNA RAPD [J]. Journal of Inner Mongolia University (NATURAL SCIENCE EDITION), 1998, 29 (6): 801-805. 